\title{
Análisis prospectivo de los escenarios posibles para para la educación superior en México, "Fuente de retos para el sistema educativo nacional"
}

\author{
Prospective analysis of possible scenarios for higher education in Mexico, "Source of challenges \\ for the national educational system" \\ Hugo A. Buitrón-Ramírez a , José F. Hernández-Gracia ${ }^{b}$, Martín Sauza-Toledo ${ }^{c}$ \\ Thelma T. Altamirano-Cardoso ${ }^{d}$,Venancio Avendaño-Hernández ${ }^{e}$
}

\begin{abstract}
:
From a prospective point of view, the currently possible scenarios for education in Mexico are considered to be a source of challenges for the national educational system, which highlights the growing demand that was projected for the higher educational level in the coming years of the this century, according to what was published in 2005 by the Center for Social Studies and Public Opinion and where scenarios that demanded a greater investment for higher education were visualized, which proposed an increase and improvement of the infrastructure of higher education, as well as achieving broader coverage of this educational level, among other challenges.
\end{abstract}

Keywords:

Prospective, scenarios, education

\section{Resumen:}

Desde un punto de vista prospectivo los escenarios posibles en la actualidad para la educación en México se consideran que son fuente de retos para el sistema educativo nacional, en los cuales destaca la demanda creciente que se proyectaba para el nivel educativo superior en los próximos años del presente siglo, de acuerd o a lo publicado en 2005 por el Centro de Estudios Sociales y de Opinión Publica y en donde se visualizaron escenarios que reclamaban una mayor inversión destinada a la educación superior, que planteaban un incremento y mejora de la infraestructura de la educación superior, así como lograr ampliación de la cobertura de este nivel educativo, entre otros retos.

\section{Palabras Clave:}

Prospectivo, escenarios, educación

\section{Introducción}

Se considera que los escenarios tienen su origen en varios factores; el primero debido a los cambios demográficos que se desarrollan en el presente siglo, como consecuencia del descenso de la tasa de natalidad en los últimos años, además del crecimiento de la población perteneciente a los grupos de mayor edad, que llegarán antes de la mitad de este siglo XXI a rebasar en proporción a la población más joven (CESOP, 2005.

De igual forma el Consejo Nacional de Población (CONAPO), expone que en el año de 1950 la edad promedio de la población mexicana era de 23.3 años y para el año 2000 el promedio de edad que se registró fue de 26.1 años, lo que representó un envejecimiento en promedio de 2.8 años. Sin embargo, afirma que en los próximos años el país envejecerá en forma acelerada, al término de la tercera década de este nuevo siglo se alcanzará una edad promedio de 36.5 años y para el 2050 alcanzará los 42.1 años. Así tenemos, que en la primera mitad de este siglo XXI la población envejecerá en promedio 16.0 años (CONAPO, 2005).

Otro de los factores, es el que enfrenta el sistema educativo nacional y de acuerdo a la Asociación Nacional 
de Universidades e Instituciones de Educación Superior (ANUIES), es el acelerado proceso de urbanización, en donde la sociedad mexicana ha pasado de ser una sociedad rural a una sociedad urbana, cuyo desarrollo se vincula al proceso de industrialización del país y de sus regiones (ANUIES, 1999), en donde las instituciones de educación superior no son ajenas a este fenómeno, ya que se ubican principalmente en centros urbanos, por lo que se dice que este nivel educativo es un fenómeno característicamente urbano. Por lo tanto, los desequilibrios manifiestos en la educación superior también responden en gran medida a este proceso de urbanización y del mejoramiento en el ingreso de las familias.

Por otra parte y con base a lo expuesto por la Presidencia de la República en 2004, otro de los factores que explican en la actualidad el aumento de la demanda de educación superior, es efectivamente el mejoramiento de los indicadores del sistema educativo nacional en los niveles previos al nivel superior. Estos indicadores son la tasa de: reprobación, absorción, deserción y de eficiencia terminal, al mejorar indicadores como la cobertura y la eficiencia terminal de un nivel educativo se genera mayor población demandante de servicios educativos de los siguientes niveles (CESU, 2002).

Otro de los factores de influencia en la gestión de la educación en México, son los montos de inversión y la distribución del gasto a nivel nacional, en ese rubro.

Según Elisa Villa (2016), en su artículo titulado ¿Cómo va la educación en México?, haciendo referencia al informe de 2015 "Education at a glance" realizado por la Organización para la Cooperación y el Desarrollo Económico (OCDE), sostiene que México es el país que más gasta en educación en donde el porcentaje del PIB destinado a la educación es cercano al $7 \%$, por arriba del $6.1 \%$ de la media de los países miembros, pero los resultados no son los esperados.

De igual forma, la autora mencionaque más del $92 \%$ del presupuesto total en educación primaria, secundaria y media superior en México se destina a remuneración personal y alrededor del $83 \%$ es destinado exclusivamente para los salarios de los maestros.

Por otra parte, otro de los factores y tema central del presente escrito, es el que está basado en los cambios que se están generando en la actualidad, en el entomo económico, político y social, como lo citaba en 2018 el Subsecretario de Educación Superior de la Secretaría de Educación Pública (SEP), Rodolfo Tuirán Gutiérrez en su artículo "La educación superior en México: avances, rezagos y retos":

Estos cambios generan enormes expectativas y seguramente, de consolidarse, tendrán efectos observables y duraderos en el entorno económico y social. La formación de técnicos, profesionales y científicos cada vez más competentes, la producción del conocimiento y la generación de innovaciones son contribuciones que la sociedad espera de sus instituciones educativas. Una educación superior pertinente y de calidad no sólo es una aspiración legítima, sino una condición fundamental para impulsar el desarrollo del país, fortalecer la ciudadanía, mejorar la competitividad y lograr una inserción ventajosa en la economía basada en el conocimiento.

Además, se percibe la necesidad de adicionarle a la educación superior, una mayor vinculación con el sector productivo y la evaluación integral del proceso educativo. Al respecto y como lo cita José Luis Chávez Chávez (2007), en su artículo "La educación en México bajo el horizonte del año 2020"

La Educación Superior en México, debe intentar responder a los patrones internacionales y la dinámica de los procesos de globalización económica y tecnológica. La competencia en los sistemas de educación superior estará cada vez más globalizada, ya no se puede hablar de alumnos de primera, segunda y tercera, por lo que se requiere transformar los procesos institucionales de manera uniforme.

Con base en lo anterior citado, se puede decir que una de las formas de responder a esos retos, es propiciando un cambio de paradigma de un modelo educativo con alcances nacionales, a un modelo que contemple la dinámica de globalización de saberes, que vayan de la mano con los cambios económicos, sociales y tecnológicos, como lo sostiene Rigoberto Lanz (2003), en su artículo "¿Qué quiere decir "reforma universitaria" ?: lo que quieren, los que quieren reformas":

La transformación institucional deberá propiciar un cambio en el paradigma del modelo educativo, de tal forma que implique el cuestionamiento del modelo social y económico en el que se fundamenta y que conduzca a las universidades a otros modos de pensar, de organizar, de producir y transmitir los saberes.

Por otra parte y desde el punto de vista legal, la Organización Mexicanos Primero (2017), señala con base al Diario oficial de la Federación (DOF) 2016, Articulo 2 que, la reciente reforma a la Ley General de Educación (LGE) señala que "Todo individuo tiene derecho a recibir educación de calidad en condiciones de equidad, por lo tanto, todos los habitantes del país tienen las mismas oportunidades de acceso y permanencia en el sistema educativo nacional".

De igual forma como lo sostiene Ángel Hernández en su artículo "Educación Superior desafío para el sistema 
educativo nacional" en la actualidad, en el Plan Nacional de Desarrollo 2019-2024, el Ejecutivo Federal asienta que en gobiernos anteriores se pretendió acabar con la gratuidad de la educación superior y se sometió a las universidades públicas a un "acoso presupuestal sin precedente". Añade que esta situación, entre otras consecuencias, provocó la exclusión de cientos de miles de jóvenes de las universidades.

En la necesidad de resolver la brecha educacional que se generó en el país, las Universidades para el Bienestar Benito Juárez García son la respuesta del actual gobiemo para revertir los rezagos en educación su perior e iniciaron actividades en marzo de 2019 con 100 planteles a nivel nacional.

\section{Conclusiones}

Expuesto lo anterior, los retos para la educación superior se pueden considerar multifactoriales, en donde los rezagos, la obligación en el cumplimiento de indicadores y las gestiones orientadas a resultados que no contribuyen al perfil de egreso necesario para que la educación impacte de manera positiva en la vida de los estudiantes, ha ocasionado que un gran porcentaje de ellos no se inserte de manera exitosa en el ámbito laboral. Por lo cual, se hace necesario implementar acciones concretas dentro del plan, que permitan mejorar los indicadores tales como la tasa de: reprobación, absorción, deserción y de eficiencia terminal, ya que, al influir en indicadores tales como la cobertura y la eficiencia terminal de un nivel educativo, se incide de forma directa en la calidad de vida del estudiante que egresa del nivel superior, que impacta al incorporarse de manera exitosa al sistema productivo nacional. Es de considerar que, aunque los seres humanos somos diferentes en muchos aspectos $y$ sumado a las diferencias que existen en los distintos niveles y sectores educativos públicos y privados, en donde se debe considerar fundamental, fortalecer la investigación referente al tema de equidad educativa, ya que, desde el punto de vista de los investigadores, es un tema que se le ha dado mediana relevancia en el ámbito educativo nacional.

\section{Referencias}

ANUIES, (1999). La Educación Superior en el Siglo XXI: Líneas estratégicas de desarrollo, una propuesta de la Asociación Nacionalde Universidades e Instituciones de Educación Superior, México. Recuperado de http://www.anuies.mx/index 1024.html

Centro de Estudios Sobre la Universidad CESU (2002). Proyecto: El Financiamiento para las Instituciones de Educación Superior en México 1990-2002. Centro de Estudios sobre le Universidad (CESU) de la UNAM; México.

Centro de Estudios Sociales y de Opinión Pública CESOP (2005). Perspectiva de la educación en México para el siglo XXI. Cámara de diputados LIX Legislatura. Recuperado de http://www3.diputados.gob.mx/camara/content/download/21139/ 104898/file/FATSE004\%20Perspectiva \%20de\%20la\%20educaci on\%20superior\%20en\%20Mexico....pdf
Chávez, Chávez José Luis (2007). La educación en México bajo el horizonte del año 2020. Revista iberoamericana de Producción Académica y gestión Educativa ISSN 2007-8412. Recuperado de https://pag.org.mx/index.php/PAG/article/download/122/169

CONAPO (2005); Proyecciones de la Población en México 2000 - 2050. Recuperado de http://www.conapo.gob.mx

Hernández, Ángel (09 de enero 2020). Educación superior desafío para el sistema educativo nacional, México; Revista Vértigo político. Recuperado https://www.vertigopolitico.com/politica/federal/educacionsuperior-desafio-para-el-sistema-educativo-nacional

Lanz, Rigoberto (2003). "¿Qué quiere decir "reforma universitaria” ?: lo que quieren los que quieren reformas", Caracas: Mimeo.

Mexicanos primero (2017). Todo estado de la educación en México 2017. P. 15, México: Editorial Mexicanos primero, visión 2030 A.C., Recuperado de http://www.consulta.mx/index.php/estudios-einvestiga ciones/otros-estudios/item/915-estado-de-la-educacionen-mexico-2017

Tuirán, Rodolfo (2018). "La educación superior en México: avances, rezagos y retos", Subsecretario de Educación Superior de la Secretaría de Educación Pública (SEP). Recuperado de http://online.aliat.edu.mx/adistancia/Calidad/unidad4/lecturas/TX T_1_S4_EDUC_SUP_AVAN_REZ_RET_TUIRAN.pdf

Villa Elisa (2016). ¿Cómo va la educación en México? El universal.com.mx. Recuperado de http://www.eluniversal.com.mx/entrada-deopinion/colaboracion/el-a punte/2016/08/4/como-va-la-educacionen-mexico 